\title{
Stability of a buoyancy-driven coastal current at the shelf break
}

\author{
By C. CENEDESE ${ }^{1}$ AND P. F. LINDEN ${ }^{2}$ \\ ${ }^{1}$ Physical Oceanography Department, Woods Hole Oceanographic Institution, \\ Woods Hole, MA 02543, USA \\ ${ }^{2}$ Department of Mechanical and Aerospace Engineering, University of California, \\ San Diego, CA 92093, USA
}

(Received 16 December 1999 and in revised form 19 June 2001)

\begin{abstract}
Buoyancy-driven surface currents were generated in the laboratory by releasing buoyant fluid from a source adjacent to a vertical boundary in a rotating container. Different bottom topographies that simulate both a continental slope and a continental ridge were introduced in the container. The topography modified the flow in comparison with the flat bottom case where the current grew in width and depth until it became unstable once to non-axisymmetric disturbances. However, when topography was introduced a second instability of the buoyancy-driven current was observed. The most important parameter describing the flow is the ratio of continental shelf width $W$ to the width $L^{*}$ of the current at the onset of the instability. The values of $L^{*}$ for the first instability, and $L^{*}-W$ for the second instability were not influenced by the topography and were $2-6$ times the Rossby radius. Thus, the parameter describing the flow can be expressed as the ratio of the width of the continental shelf to the Rossby radius. When this ratio is larger than 2-6 the second instability was observed on the current front. A continental ridge allowed the disturbance to grow to larger amplitude with formation of eddies and fronts, while a gentle continental slope reduced the growth rate and amplitude of the most unstable mode, when compared to the continental ridge topography. When present, eddies did not separate from the main current, and remained near the shelf break. On the other hand, for the largest values of the Rossby radius the first instability was suppressed and the flow was observed to remain stable. A small but significant variation was found in the wavelength of the first instability, which was smaller for a current over topography than over a flat bottom.
\end{abstract}

\section{Introduction}

Buoyancy-driven coastal currents occur in many parts of the world's oceans. The outer edges of these currents are bounded by fronts where the interface, which slopes upwards from the coast, intersects the surface. Particularly striking examples are the Leeuwin Current (Griffiths \& Pearce 1985; Pearce \& Griffiths 1991), the Norwegian Coastal Current (Johannessen \& Mork 1979) and the East Greenland Current (Wadhams, Gill \& Linden 1979). The currents owe their existence to the presence of a coast. Since there is no flow normal to the coast, there is no Coriolis force parallel to the coast. Motion can occur parallel to the coast accompanied by a Coriolis force directed normal to the coast. Hence the resulting current hugs the coastline with the coast on its right looking downstream (in the northern hemisphere). 
Other examples are fresh water entering the ocean from rivers such as the Amazon basin, or water masses passing through straits from one basin to another, such as between the Atlantic Ocean and the Mediterranean sea through the Strait of Gibraltar. These flows are often observed to turn right (in the northern hemisphere) to form coastal currents.

Satellite images of these currents show that they are unstable, giving rise to meanders and the detachment of eddies from the main current (see figure 5 of Legeckis 1978 or figure 12 of Wadhams et al. 1979). The edges of these currents represent boundaries between different water properties and the stability of the front greatly affects the rate of mixing across the front, as well as the effectiveness of the current in transporting heat or salinity along the coast. Detached eddies (with scales of 10 to $10^{2} \mathrm{~km}$ ) can transfer relatively large volumes of water across the front. The growth of waves increases the length of the lateral boundary between different water masses, promoting mixing by small-scale processes such those described by Joyce (1977).

The behaviour of a buoyant current over a flat bottom was extensively investigated by Griffiths \& Linden (1981, hereafter referred to as GL) and reviews on fronts and eddies can be found in Griffiths (1986) and Linden (1991). Buoyancy-driven currents over a flat bottom become unstable to non-axisymmetric disturbances. The wavelength and phase velocities of the disturbances observed by GL agree with their baroclinic instability model of two-layer flow, which takes frictional dissipation caused by Ekman layers into account.

The coastal currents described above often flow above the shelf break, where the relatively flat continental shelf gives way to the steeper continental slope. The characteristics of the continental slope vary in different parts of the world ocean and can range from a gentle slope to a steep ridge. A well-documented case of the outer front of a coastal current lying above the shelf break is the front in the Middle Atlantic Bight. This front extends from Nova Scotia to Cape Hatteras, where the warm and saline waters from the North Atlantic Ocean encounter the cooler, fresher waters of the continental shelf (Wright 1976; Linder \& Gawarkiewicz 1998). Shelf-break eddies of diameter $40 \mathrm{~km}$ at times contort the front (Garvine et al. 1988; Houghton, Olson \& Celone 1986). The existence of eddies and meanders on a scale of about $50 \mathrm{~km}$ was also observed on the polar front in the Iceland-Faroe Ridge area (Hansen \& Meincke 1979; Hallock 1985). These observations motivated us to investigate the effects of bottom topography on the behaviour and the stability of a buoyancy-driven coastal current.

The baroclinic stability of a front over steep topography was investigated using a geostrophic momentum approximation by Flagg \& Beardsley (1978) who performed a perturbation analysis of Orlanski's (1968) nonlinear eigenvalue model. Their results showed the existence of unstable modes over a finite bottom slope, but their characteristic e-folding times increased from 2-5 days over a flat bottom to 50 days over steep topography. An extension of this model investigating a larger wavenumber range by Gawarkiewicz (1991) examined the stability of inviscid frontally trapped waves along a shelf break both for a winter and a summer front configuration for parameters typical of the front south of New England. A further difference between this two-layer model of a geostrophically balanced front and Flagg \& Beardsley (1978) was the presence of an unbounded bottom topography offshore beyond the frontal region. The results obtained with parameters characteristic of the winter in the Middle Atlantic Bight showed that the most unstable wave had an e-folding growth time scale of 9.5 days, a wavelength of $25 \mathrm{~km}$ and was surface trapped. This 
growth rate is faster and the most unstable wavelength is approximately $20 \%$ more unstable, than found by Flagg \& Beardsley (1978). A recent two-layer baroclinic frontal geostrophic model by Swaters (1993) has been applied to investigate the behaviour of a buoyancy-driven coastal current over topography. It is found that a bottom that slopes in the same sense as the fluid interface retards the growth of perturbations (Reszka \& Swaters 1999a), while a bottom that slopes in the opposite sense to the frontal interface enhances the instability (Reszka \& Swaters 1999b). The latter configuration was applied to the Gaspé Current, an intense buoyancy-driven jet in the western Gulf of St. Lawrence. Reszka \& Swaters (1999b) found that the dominant wavelength of the resulting instability was $60 \mathrm{~km}$ with e-folding times of 4-5 days, consistent with observations. Assuming that for the basic current the potential vorticity gradient due to relative vorticity is negligible, these results agree with the necessary condition for instability found by Pedlosky (1964) for a quasi-geostrophic two-layer system. Whenever the bottom and the front slope in opposite directions the gradients of the potential vorticity or thickness of each layer are of opposite sign, satisfying this necessary condition. However, it must be noted that this is a necessary, not a sufficient, condition. Therefore, there can be cases where bottom topography that slopes in the opposite direction from the frontal interface can decrease the growth rate and amplitude of frontal instability as shown by Flagg \& Beardsley (1978). The present understanding of the effects of topography on the stability of a front is still incomplete, as all of the previous theoretical and numerical works present different situation-specific hypotheses. Therefore, we do not have a universally applicable description of the conditions under which topography stabilizes or destabilizes a front.

The work described in this paper aims to investigate the influence of bottom topography on the stability of a buoyancy-driven surface current or its outer front through laboratory experiments. Similar experiments have been performed before by Condie (1993) and compared with an analytical, inviscid two-dimensional model. Condie's results show a strong stabilizing effect of steep topography. The new aspects of the experiments described in this paper are the investigation of the current at later times after the appearance of the first instability and the different geometry of the bottom topography that simulates a continental slope, a continental ridge (shelf break) and a combination of the two (figure 1). Typical examples of these geometries occur in the ocean; the continental ridge (figure $1 b$ ) is similar to the Iberian Peninsula where there is an almost vertical descent from a narrow shelf to the ocean bottom; the sloping bottom topography (figure $1 c$ ) is similar to the shelf west of Ireland and the combination a continental ridge followed by a gentle sloping bottom (figure $1 d$ ) can be found in the Middle Atlantic Bight. In these experiments the instability is likely to be predominantly baroclinic since the fractional depth of the currents is not small (e.g. Killworth 1980; Holland \& Haidvogel 1980).

The experimental apparatus is described in $\S 2$. In $\S 3$ we review the instability of a buoyancy-driven current over a flat bottom. The behaviour of currents with a small (large) Rossby radius of deformation over different topographies is discussed in $\S 4$ (§5). In $\S 4$ we also present new results on the behaviour of a buoyancy-driven current over a flat bottom at later times. A comparison between the results for a flat bottom and topography is presented in $\S 6$, and a comparison of the results for the different topographies is given in $\S 7$. Finally the conclusions of the work are discussed in $\S 8$.

\section{The experiments}

A sketch of the apparatus is shown in figure 1. The experiments were conducted in a transparent Perspex tank of depth $30 \mathrm{~cm}$ with a circular base of radius $R=45 \mathrm{~cm}$. 

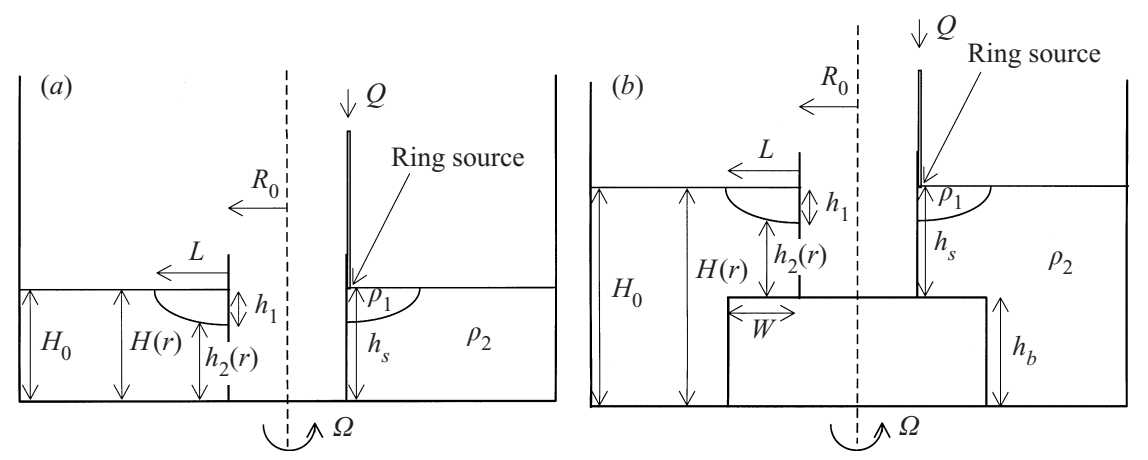

(c)

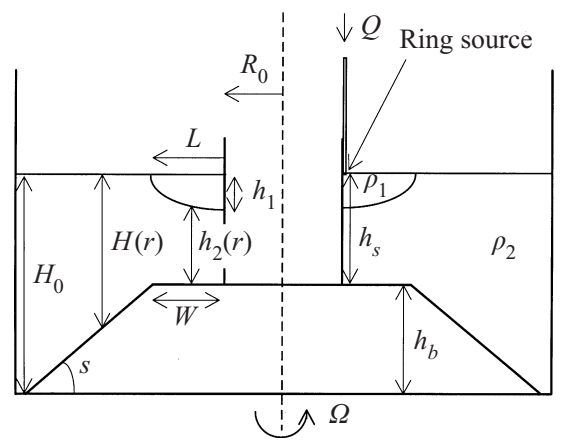

(d) $\quad \stackrel{R_{0}}{\longleftarrow} \downarrow Q \quad$ Ring source

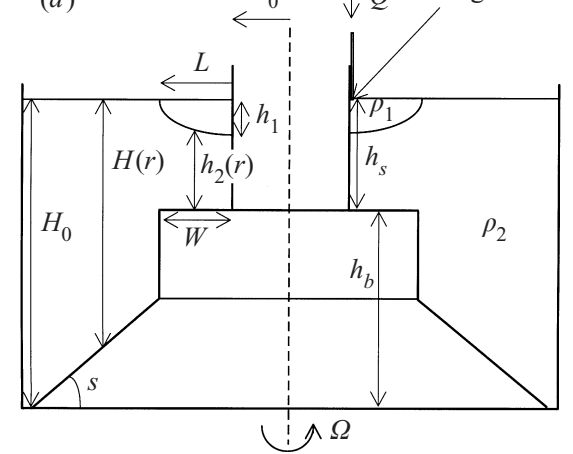

FIGURE 1. Sketches of the experimental apparatus for $(a)$ the flat bottom case, $(b)$ step topography, $(c)$ slope topography and $(d)$ step over a slope topography.

This was mounted concentrically on a $1 \mathrm{~m}$ diameter, rotating turntable with a vertical axis of rotation. The vertical boundary against which the currents were generated was a Perspex cylinder of radius $R_{0}=13 \mathrm{~cm}$, also concentric with the axis of rotation so that the flow was confined in an annular region $32 \mathrm{~cm}$ wide. Currents with small Rossby radius were observed to become unstable within approximately one-half the width of this region, and therefore the presence of the outer boundary is unimportant for the problem of main interest in this paper. However, the evolution of the flow at later times as well as currents with large Rossby radius were influenced by the presence of the outer boundary. Further studies in a larger tank are necessary to completely understand these flows.

Four different bottom topographies were adopted:

flat topography (figure 1a);

step topography (figure $1 b)$, where a cylinder $(20 \mathrm{~cm}$ radius and $9.5 \mathrm{~cm}$ deep) was positioned underneath the vertical boundary against which the currents were generated;

slope topography (figure $1 c$ ), where a cylinder $(20 \mathrm{~cm}$ radius and $9.5 \mathrm{~cm}$ deep) was sealed to an axisymmetric transparent sloping bottom;

step over a slope topography (figure $1 d$ ), where a cylinder $(20 \mathrm{~cm}$ radius and $9.5 \mathrm{~cm}$ deep) was positioned over another cylinder $(20 \mathrm{~cm}$ radius and $9.5 \mathrm{~cm}$ deep) sealed to an axisymmetric transparent sloping bottom.

Hereafter, we will refer to the region of width $W=7 \mathrm{~cm}$ over the truncated cylinder as the continental shelf, the edge of this plastic cylinder as the continental ridge, and the sloping topography as the continental slope. 
The tank was filled with a salt solution of density $\rho_{2}$ to a maximum fluid depth at the tank wall $H_{0}$, and was brought to solid-body rotation. A reservoir $R_{1}$ of fresh dyed water of density $\rho_{1}<\rho_{2}$ was connected to a source via a plastic tube. A constanthead, gravity-fed source was achieved by placing the reservoir $\left(R_{1}\right)$ on the top of the rotating table, and gave a constant flow rate $Q$ through the tube, monitored by a flow meter. The source consisted of a ring of copper tubing containing many small holes and covered with plastic foam and was positioned at the free surface, adjacent to the cylindrical boundary. This source fitted snugly around the inner cylinder in a horizontal plane and introduced the surface buoyant fluid almost uniformly around the whole circumference of the cylinder. The currents were produced at the free surface to avoid a significant radial loss of fluid in the strong Ekman layers that form at the rigid horizontal boundaries.

The buoyancy forces are described by the reduced gravity $g^{\prime}=g\left(\rho_{2}-\rho_{1}\right) / \rho_{1}$, where $g$ is the gravitational acceleration. In the experiment $g^{\prime}$ took values between $0.90 \mathrm{~cm} \mathrm{~s}^{-2}$ and $4.50 \mathrm{~cm} \mathrm{~s}^{-2}$ and the Coriolis parameter $f=2 \Omega$ was varied from $0.6 \mathrm{~s}^{-1}$ to $5.0 \mathrm{~s}^{-1}$. The flow rate through the circular source was held constant at $Q=10 \mathrm{~cm}^{3} \mathrm{~s}^{-1}$. The continental slope was fixed at $s=\tan \alpha=0.5$, where $\alpha$ is the angle between the slope and the horizontal, and the depth $h_{s}$ of the water below the source was either $3.5 \mathrm{~cm}$ or $7.0 \mathrm{~cm}$. The Rossby radius of deformation, $R_{D}=\sqrt{g^{\prime} h} f^{-1}$, was based on the upper-layer-current maximum depth $h_{1}^{*}$ at the wall (figure 1) immediately prior to the appearance of growing disturbances. (Note that in cases where the currents undergo two instabilities there will be two Rossby radii of deformation, each one based on the current depth measured immediately prior to the appearance of the respective instability). We use a chronological definition for the instabilities observed, where the first to appear is named the 'first instability' and the second to appear is named the 'second instability'. A table containing an overview of the experimental parameters and measured quantities is available from the JFM editorial office.

A video camera was mounted above the tank and fixed to the turntable so that velocity measurements were obtained in the rotating frame. A computer system with a frame-grabber card and the image processing software DigImage (Dalziel 1992) was used to acquire and process the images from the video records of the flow. The current was made visible by dying the source water with food colouring and by adding buoyant paper pellets on the surface. The motion of dyed current was observed both from top and side views. The current depth was determined by eye from the side view and, for some experiments, by measuring the reduction in light intensity transmitted through the dyed layer during the experiment and comparing it to that through the top dyed layer with no flow at the beginning of the experiment (for details see Cenedese \& Dalziel 2000). In order to do this with the camera positioned above the tank, light sources were placed under the tank. In fact this technique, also referred to as a measurement of optical thickness, gives a measure of the average concentration of the dye over the whole fluid thickness. Since only one layer is dyed, with a uniform concentration of dye, the measure of the optical thickness gives the depth of the top dyed layer. We used food colour with a concentration of $1 \mathrm{ml}^{-1}$. The software 'DigImage' (Dalziel 1992) was used to determine intensity fields, to manipulate the recorded images and to find the intensity contours of the front in order to calculate its depths and width. In order to measure depth fields over the (opaque) plastic cylinder, lights were positioned around the tank and switched on only during the early stages of the experiment until the dyed fluid spread over the edge of the cylinder. The optical thickness method did not work quantitatively in this case, and depth (width) measurements were taken by eye from the side (top) view. Throughout an experiment 
the surface current width $L$ and the maximum current depth $h_{1}$ at the wall were measured. Hence, knowing the total fluid depth $H(r)$, as a function of the distance $r$ from the tank centre, (figure 1), the depth of the lower layer $h_{2}=H\left(R_{0}+L\right)-h_{1}$ and the ratio of layer depths $\gamma=h_{1} / h_{2}$ were determined at the front $\left(r=R_{0}+L\right)$. Rigorously, $H\left(R_{0}+L\right)$ represents the total fluid depth at the radius where the front intersects the free surface. However, we are interested in the lower-layer depth at the point of maximum radius $r$ where the buoyant current depth is approximately $h_{1}$. We, therefore, make an approximation in deriving $h_{2}$ and consider the total fluid depth to be the same across the whole front and equal to $H\left(R_{0}+L\right)$. This approximation is justified by a combination of the facts that the front is quite steep and narrow compared to the bottom slope.

The velocities were measured by tracking the paper pellets on the surface using DigImage. Approximately 400 paper pellets were located in each frame and the velocities were obtained by sampling the video film at a frequency of $2.5 \mathrm{~Hz}$. Particle velocities were calculated over 5 samples and the velocity field was obtained by mapping the individual velocity vectors onto a rectangular grid using spatial averaging over $10 \mathrm{~cm}$, and temporal averaging over $0.4 \mathrm{~s}$. The vorticity was calculated from these gridded velocity data.

Unfortunately, in order to measure the fluid thickness and the velocity field for the same experiment we were not able to resolve the velocity fluctuations due to the instability within the boundary current accurately. However, we were able to obtain the velocity of the basic boundary current.

\section{Instability of a buoyant current over a flat bottom}

The effect of the buoyancy force caused the lighter fluid from the source to move radially outward. Hence, the lighter fluid started moving anticyclonically in order to conserve angular momentum. Vortex tubes in the ambient fluid beneath the buoyant current were compressed and anticyclonic flow was also set up in the lower layer, the magnitude of which depended on the ratio of layer depths. The Coriolis force difference resulting from this azimuthal shear across the interface was balanced by the pressure force due to the sloping density interface. The buoyant current depth decreased away from the inner wall until it intersected the free surface at a distance $L$. The maximum depth $h_{1}$ of the buoyant current was observed at the inner wall, and the distance $L$ represents the width of the current.

The behaviour of a buoyant current over a flat bottom was investigated by GL. They observed that a constant flow rate of buoyant fluid produces an upper-layer current having a depth and width increasing as $t^{1 / 2}$ (see figure 2 in GL). Although this zonal current is time dependent, the time evolution is slow compared to $f^{-1}$ and it may be regarded as being quasi-steady and in quasi-geostrophic balance as described above. This approximation is justified by the small flow rate used both in GL and our experiments. GL observed a wave-like disturbance at the interfacial front that ultimately grew to large amplitude, and they developed a model for baroclinic instability with friction that successfully described the observed instability. The dominant wavelength of such disturbances and the current width at the onset of the instability scale with, and are a few times larger than, the Rossby radius of deformation of the upper layer.

Initially, the observed disturbances on the current were everywhere anticyclonic. Cyclonic relative vorticity, initially concentrated at the outer edge of the current, moved behind the crests of the waves and produced regions of cyclonic motion at the 
(a)

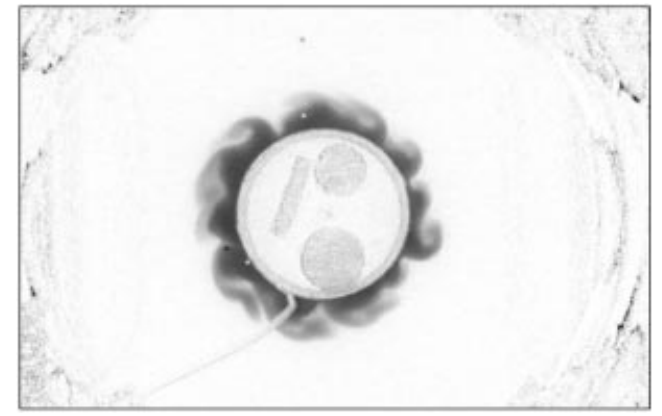

(c)

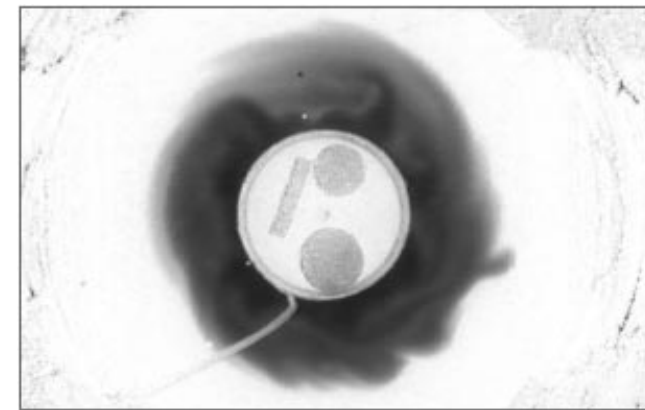

(b)

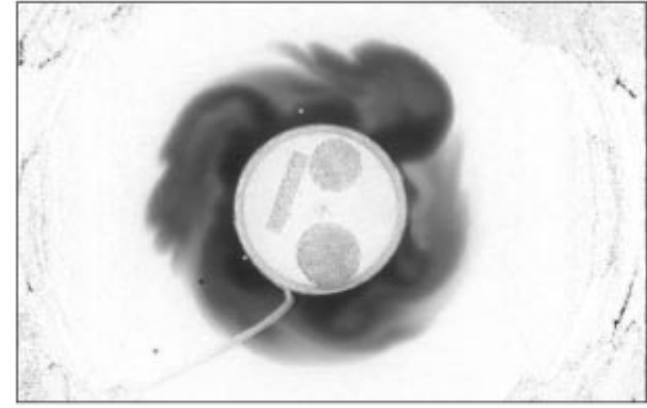

(d)

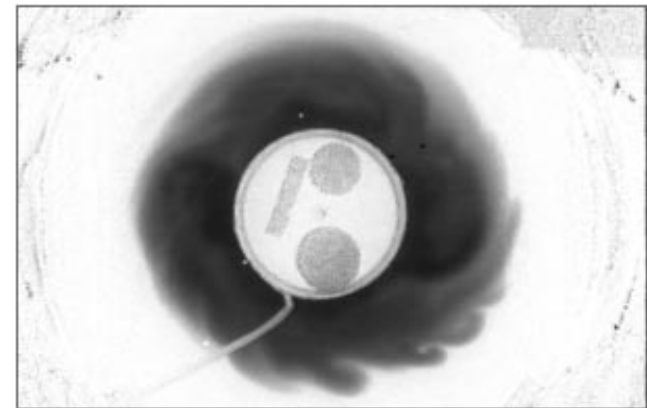

FIGURE 2. Flat topography. A sequence of plan view images (taken in the rotating frame) showing a buoyant current produced by the ring source which is unstable to wave-like disturbance. The images were taken at $(a)$ 29, (b) 143, (c) 187 and (d) 300 revolutions after the source has been activated. The direction of rotation is anticlockwise and the current and the waves are travelling anticyclonically (i.e. clockwise). $f=3.0 \mathrm{~s}^{-1}, g^{\prime}=2.2 \mathrm{~cm} \mathrm{~s}^{-2}, Q=10 \mathrm{~cm}^{3} \mathrm{~s}^{-1}$ and $h_{s}=3.5 \mathrm{~cm}$.

troughs. The cyclonic vortices entrained fluid from the waves, which broke 'backwards' relative to the direction of the current (figure $2 a$ ). They also spread radially, increasing the mean radius of the non-axisymmetric current (figure $2 b$ ). Following this initial stage the non-axisymmetric disturbances stopped growing after reaching a certain large amplitude, and as previously observed by GL, the resultant eddies did not detach from the zonal current.

GL investigated the stability of a boundary current when both frictional dissipation due to Ekman layers and short waves are included. A previous model by Jones (1977) was used to investigate the stability of an inviscid boundary current for the limit of very long waves. For inviscid flow, all wavenumbers $K^{2}<2 F_{1}$, where $F_{1}=f^{2} L^{2} / g^{\prime} h_{1}$ is the upper-layer Froude number, are unstable while viscous dissipation stabilizes waves beyond a long-wave cutoff (see figure 6 in GL). For each value of the ratio of layer depths, $\gamma=h_{1} / h_{2}$, the minimum of the curve of marginal stability is at $K^{2}=k_{m}^{2}+l^{2}$, where $l$ is the cross-stream wavenumber and $k_{m}$ is the most unstable zonal wavenumber. Hence, the first waves that become unstable as the vertical shear $U_{s}=U_{1}-U_{2}$ increases (where $U_{1}$ and $U_{2}$ are the velocities in the upper and lower layers, respectively) have a wavelength $\lambda=2 \pi L / k_{m}$. The critical shear $U_{c}$ that must be exceeded before any wavenumber can grow increases as $\gamma$ becomes small and, for small but finite $\gamma, U_{c}$ becomes infinite, characterizing where the long-wave and short-wave cutoffs join. Therefore, there is no wavenumber that can grow below this value of $\gamma$. The critical shear becomes infinite at a value of $\gamma$ that depends on $F_{1}$. 


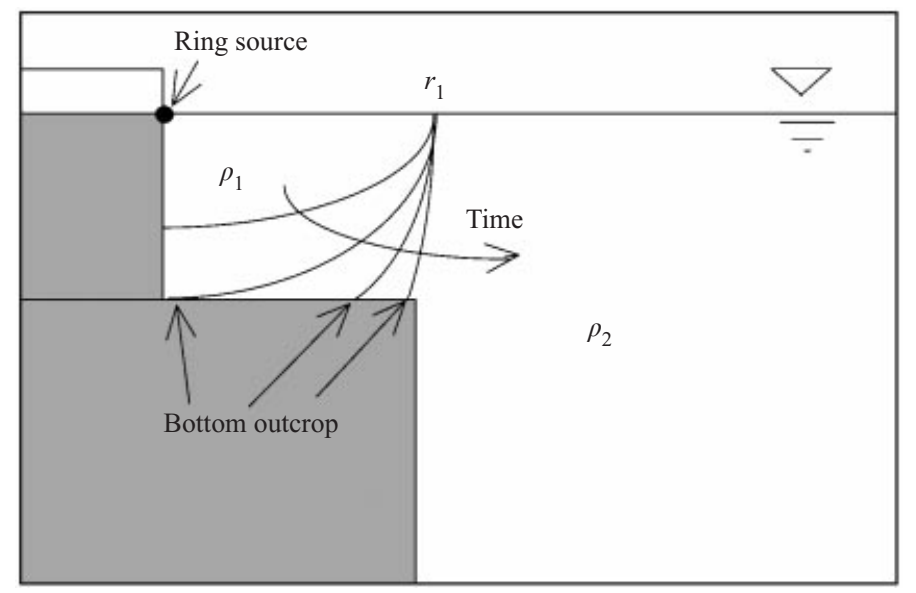

FIGURE 3. Sketch illustrating the radial movement of the density front over the continental shelf for the continental ridge topography. The bottom outcrop was observed to move radially outwards in time.

Hence, for a fixed value of $\gamma$ the flow is stable to baroclinic disturbances when the upper-layer Froude number is below a critical value $F_{c}$. In particular, for decreasing values of $\gamma, F_{c}$ increases (see figure 7 in GL). In all the experiments in GL, $F_{1}$ reaches values above $F_{c}$ a short time (approximately $30 \mathrm{~s}$ ) after the light fluid enters the tank, and the disturbances are noticeable at a Froude number $F_{1} \gg F_{c}$.

In this study we conducted experiments over a flat bottom similar to those of GL, looking in particular at the behaviour of the current after the initial stage described above. We then investigated the effects of topography. The effects of three different topographies on the flow will be described in the next two sections for small ( $(4)$ and large $(\S 5)$ values of the Rossby radius of the first instability.

\section{Instability over bottom topography for small Rossby radius}

For small Rossby radius and a flat bottom, the first instability was similar to that observed by GL as described in $\S 3$. At later times the eddies formed during the first instability remained attached to the current; interactions were observed and their vorticity decreased with time leading to an almost axisymmetric front (figure $2 c$ ). Within the current, anticyclonic vortices were still visible both from the side and top view as shown by the darkest regions in figure $2(d)$. There appeared to be little (if any) mixing, and therefore change in density, during the instability and subsequent eddy interactions. The depth and width of the current increased with time and the front of the current eventually reached the outer wall. No subsequent non-axisymmetric disturbances on the outer front were observed, even though the vortices remained in the interior until the outer wall was reached. When bottom topography was introduced for small values of the Rossby radius of the first instability, the current width $L$ at the onset of the first instability was small compared with the width $W$ of the continental shelf. The first instability, therefore, occurred within the width of the shelf region and was not affected by the presence of topography. However, the further development of the flow was modified in different ways by the three different bottom topographies. 
(a)

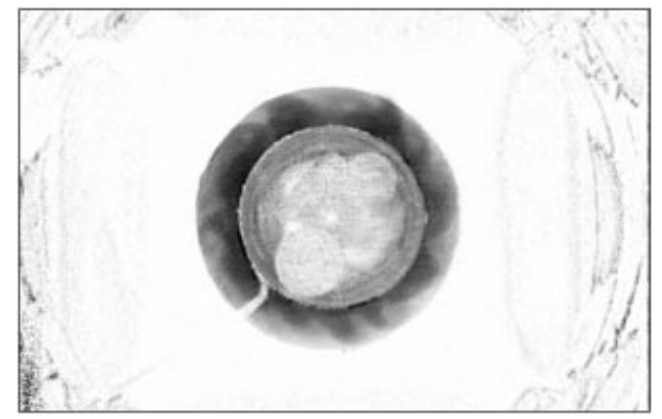

(c)

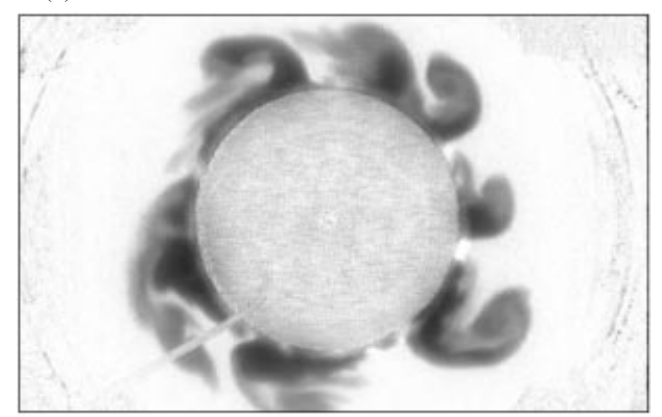

$(b)$

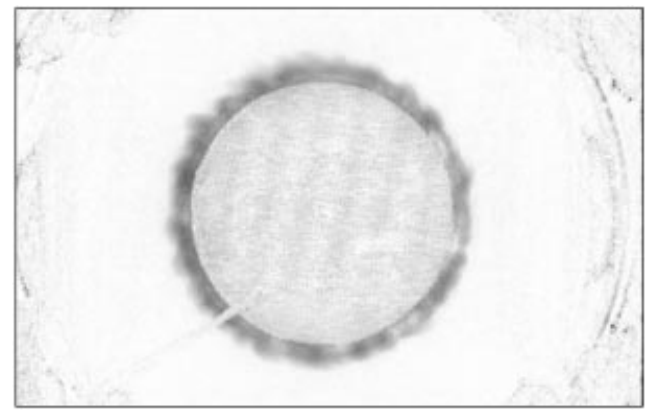

$(d)$

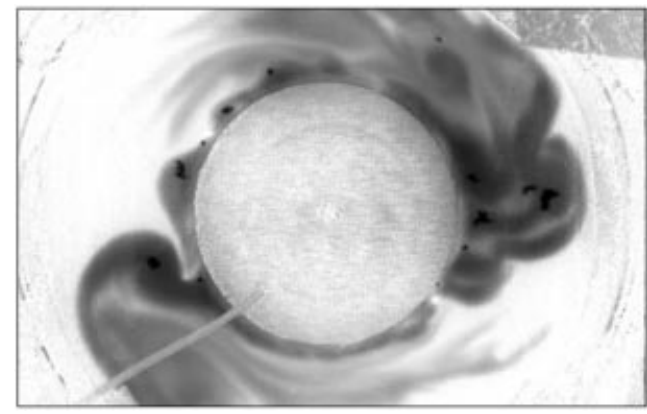

FIGURE 4. Continental ridge. A similar sequence as in figure 2 except that the current is produced over a continental ridge. The flow over the continental shelf is visible only in $(a)$, in the other images the flow is only visible beyond the continental ridge. The images were taken at the same times as in figure 2 and the variables values are the same as in figure 2 . The depth of the ridge was $h_{b}=9.5 \mathrm{~cm}$.

\subsection{The continental ridge}

The presence of a continental ridge (figure $1 b$ ) inhibited the radial spreading of the buoyant current after the first instability and constrained the flow to remain approximately over the continental shelf region within a radius $r_{1} \approx\left(R_{0}+W\right)$ (figure 3). After the first instability, eddies formed in the flow as described in $\S 3$. The 'backwards' breaking waves did not spread radially as in the flat bottom case. Instead they followed the ridge edge with an alongshore speed smaller than the inner part of the current (figure $4 a$ ), forming an approximately axisymmetric front for the current. The eddies in the current interacted and decayed while the current depth increased, and the current width was almost constant at $L \approx\left(r_{1}-R_{0}\right)$. Since the light fluid was effectively trapped on the shelf, the depth of the upper layer increased and the density interface eventually touched the bottom over the continental shelf.

After the upper layer reached the bottom, the radius of intersection of the density interface with the free surface remained fixed at $r \approx R_{0}+W$ while the position of the intersection with the bottom topography, from now on called the bottom outcrop, moved radially outwards until it reached the edge of the continental shelf as shown in figure 3. As soon as the bottom outcrop formed, dyed buoyant fluid started flowing into the bottom Ekman layer and into the Stewartson (1957) layer on the vertical wall of the continental ridge. This latter layer was approximately $1 \mathrm{~cm}$ thick and from the side view the dyed fluid was observed to descend along the vertical wall to a maximum depth of approximately $5 \mathrm{~cm}$ (usually half the depth of the wall). The dyed 
(a)

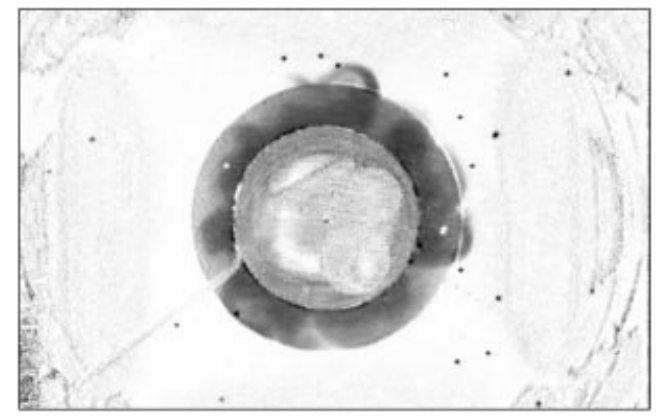

(c)

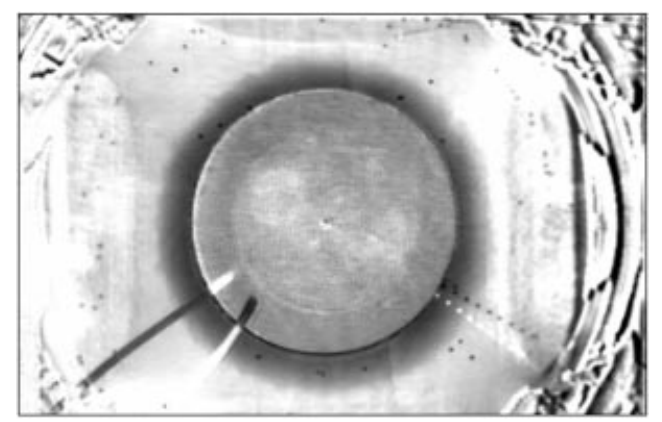

$(e)$

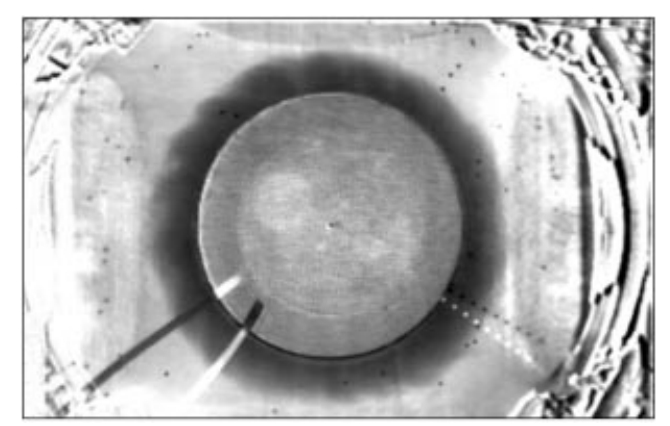

(b)

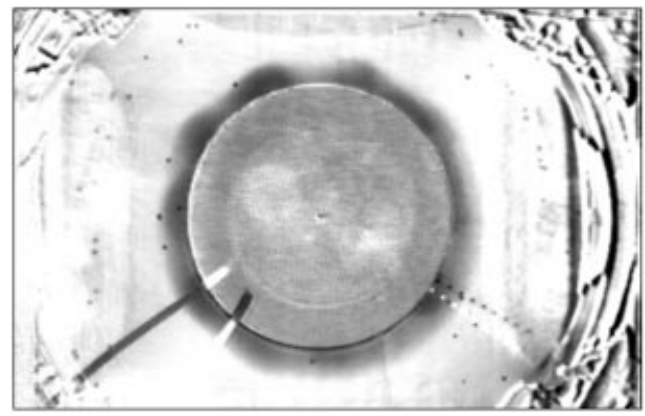

(d)

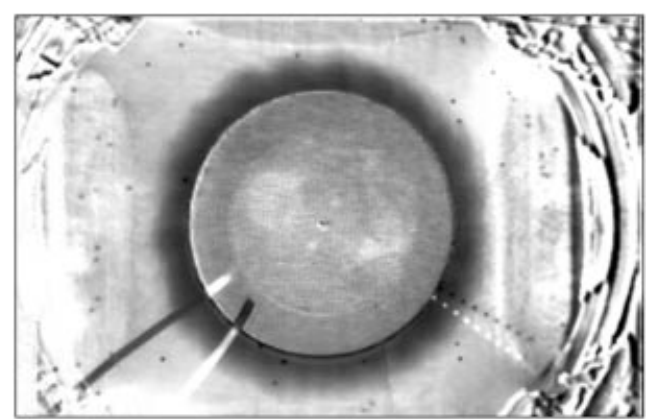

$(f)$

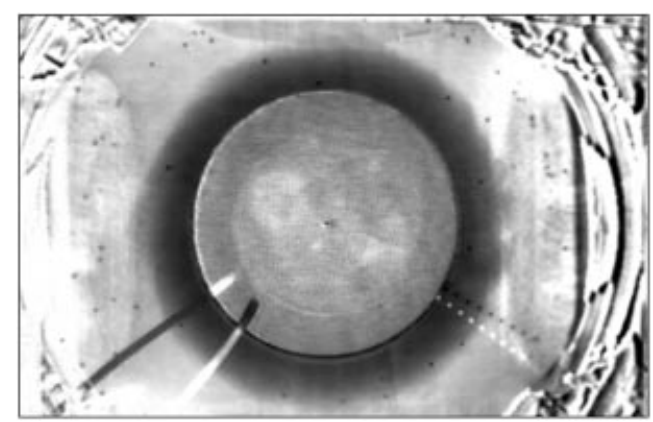

FIGURE 5. Continental slope. A similar sequence as in figure 4 except that the current is produced over a continental slope. The first four images were taken at the same times as in figure 2 and $(e)$ and $(f)$ were taken after 273 and 316 revolutions, respectively. The variables values are the same as in figure 4. The slope of the topography was fixed at $s=\tan \alpha=0.5$, where $\alpha$ is the angle between the slope and the horizontal and the continental shelf was positioned at a high $h_{b}=9.5 \mathrm{~cm}$.

fluid was never observed to reach the bottom of the tank: possibly buoyancy forces inhibited further downward motion. At this stage the ambient fluid that was initially over the continental shelf had been displaced into the deeper environment by the buoyant fluid of the current that was continuously supplied by the source. Although the step topography implies a rapid change in depth with consequent generation of vertical velocities and therefore non-hydrostatic effects in this transition adjustments, these transitions are very slow and the associated vertical velocities or non-hydrostatic effects can be neglected.

Two possible mechanisms could allow this displacement of ambient fluid. It could simply be displaced off the shelf from a shallow to a deep environment in an inviscid 


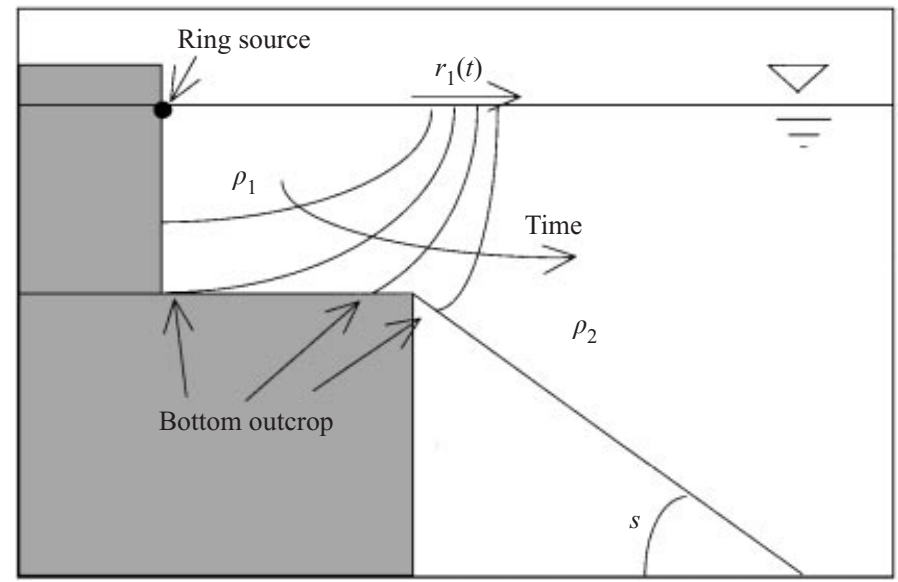

FIGURE 6. Sketch illustrating the radial movement of the density front over the continental shelf for the continental slope topography. The bottom outcrop was observed to move radially outwards in time.

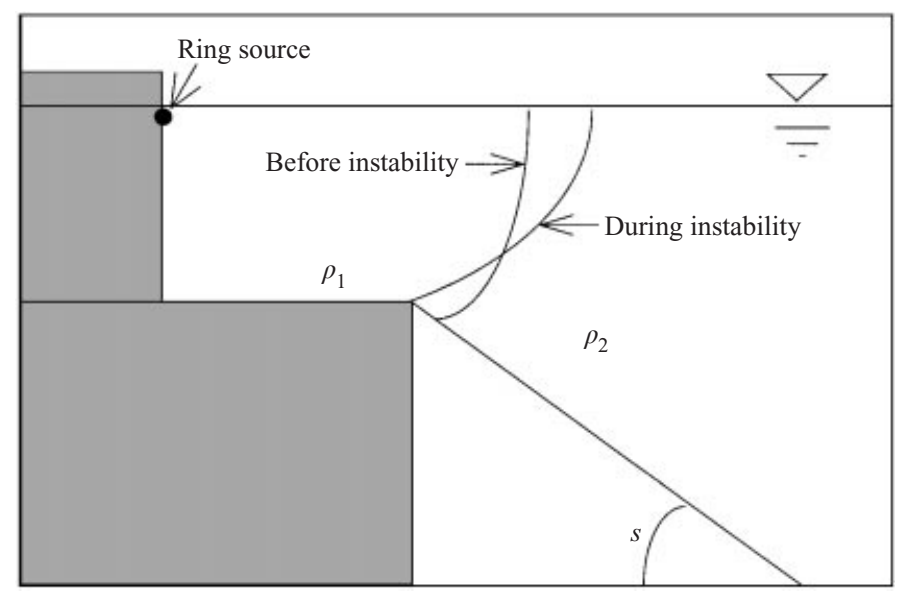

FIGURE 7. Sketch illustrating the movement of the density front before and during the second instability for a current over a continental slope.

process, in which case the ambient fluid columns must increase their relative vorticity to conserve potential vorticity. In our experiments the absence of any observed strong increase of relative vorticity in the ambient fluid immediately after it is displaced from the shelf (figure 4) suggests that this was not the case. Another possible mechanism involves the displacement of ambient fluid from the continental shelf through the bottom Ekman layer and the vertical Stewartson layer. The ambient water columns over the shelf must be compressed by the deepening overlying layer, and hence must flow in the clockwise azimuthal direction. The bottom Ekman layer flux is therefore directed offshore. The observation of dyed buoyant fluid in these layers suggests that this mechanism continued, at least to some extent, when the buoyant dyed fluid replaced the ambient fluid over the continental shelf. The net transport $T_{E}$ (per unit length) of fluid in the bottom Ekman layer transverse to the main flow is given by

$$
T_{E}=\frac{1}{2} U \delta,
$$


where $\delta=(2 v / f)^{0.5}$ is the Ekman layer thickness ( $v$ being the viscosity), and $U$ is the interior velocity. In order to conserve mass over the shelf, the net transport in the Ekman layer must balance the inflow of buoyant fluid from the source giving $T_{E}=Q / 2 \pi R_{0}$ and with (1) it is then possible to obtain $U$. The interior velocity magnitude depends upon the ratio of layer depths and, at the onset of the first instability, it is expected to be of the same order of magnitude as the velocity in the buoyant current. For the typical experimental values of $R_{0}=13 \mathrm{~cm}$, $Q=10 \mathrm{~cm}^{3} \mathrm{~s}^{-1}, f=3 \mathrm{~s}^{-1}$ and $v=10^{-2} \mathrm{~cm}^{2} \mathrm{~s}^{-1}$ (1) gives a value $U=3 \mathrm{~cm} \mathrm{~s}^{-1}$ of the same order of magnitude as the measured azimuthal velocity of the buoyant current. This result supports the possibility that ambient fluid can be displaced from the continental shelf into the deeper environment through the bottom Ekman layer and it is consistent with previous results on the dynamics of a surface-to-bottom density front on a sloping continental shelf (Chapman \& Lentz 1994, Yankovsky \& Chapman 1997).

For the few experiments in which a deeper shelf $h_{s}=7 \mathrm{~cm}$ was used, the density interface did not touch the shelf bottom but, instead, a buoyant current approximately as wide as the continental shelf formed. In all experiments, this wider current was generated by interaction of the eddies formed during the first instability and consequent reduction of their vorticity. Eventually a second instability started with a wave-like disturbance on the density interface and, in an analogous fashion to the previous instability, the disturbance grew to large amplitude. At the beginning the motion was everywhere anticyclonic (figure $4 c$ ), and the cyclonic motion that accumulated behind the wave crests immediately after the perturbation started was weaker than in the flat bottom case. This behaviour is consistent with the columns of ambient fluid (displaced by the wave-like perturbation) encountering the continental ridge and therefore not being able to stretch and acquire cyclonic motion. The subsequent flow development was different to the flat bottom case (compare figures 2 and 4) where, after the waves grew to finite amplitude, the eddies formed during the first instability did not leave the current, but started interacting and their vorticity decreased leading to an almost axisymmetric front (figure $2 c$ ). In the continental ridge case, after the second instability the front remained asymmetric (figure 4c) until it reached the outer wall. Also, in the ridge case, the front reached the outer wall in a shorter time than in the flat bottom case.

After growing to large amplitude, the anticylonic vortices in the annular region between the inner shelf and the outer wall did not separate from the remaining current and were not equally spaced along the current front. Rather, they formed new fronts roughly orthogonal to the continental ridge propagating anticyclonically as shown in figure $4(d)$. The buoyant fluid upstream of these new fronts was as wide as the continental shelf and new disturbances kept growing on that part of the current (figure $4 d$ ). The breaking-backwards waves eventually acquired cyclonic motion and carried dyed buoyant water behind the fronts. This fluid merged with the current and, therefore, we observed large azimuthal variations of the current width as shown in figure $4(d)$. The presence of these new fronts orthogonal to the continental ridge and moving anticyclonically may be influenced by the presence of the outer wall. This aspect of the flow needs further investigation, possibly with a larger tank.

In conclusion, in the presence of a continental ridge, the current passed through two instabilities, the first one when the current was over the continental shelf and the second when the current moved over the continental ridge. 


\subsection{The continental slope}

The behaviour after the current reached the edge of the continental shelf was completely different when we introduced a continental slope (figure $1 c$ ). The radial spreading of the current was not slowed down so dramatically as in the continental ridge case and the breaking waves were observed to move outwards over the continental slope. They then turned gently alongslope (figure $5 a$ ) so that the current again acquired an almost axisymmetric shape, with eddies interacting within it (figure $5 b$ ). At this stage the intersection of the density interface with the free surface kept moving slowly radially outwards while the density interface eventually touched the bottom over the continental shelf. The bottom outcrop eventually reached the continental slope and moved over it as shown in figure 6. At later times the front of this new broader current was perturbed by a wave-like disturbance (figure $5 d, e$ ). However, this instability never grew to as large an amplitude as in the continental ridge case (figure 4c), and was eventually damped and the axisymmetric current reestablished (figure $5 f$ ). During the disturbance the motion at the front was mainly anticyclonic and a streamer of upper-layer water from each wave crest stretched backwards to the neighbouring wave crest, forming an almost axisymmetric front alongslope. While the current was unstable the bottom outcrop migrated upslope, reaching the edge of the continental shelf but, as soon as the instability was suppressed, it again moved downslope to the previous position, as shown in figure 7 . Therefore, the density interface reached its maximum steepness just before the instability and then became less steep while the instability developed. When this second instability occurred, there were additional (third, fourth, fifth...) instabilities caused by the same mechanism before the current reached the outer wall. The movement of the front was clearly visible from the side view of the experiments. However, due to mixing occurring near the bottom outcrop causing the front to be less sharp, and to the small lengthscale of the front movement (a few centimetres) it was not possible to resolve the changes in interfacial slope quantitatively with the present technique. Over the continental slope the second instability occurred much later than in the continental ridge case. This behaviour seems to suggest that the presence of a continental slope reduced the growth rate and amplitude of the most unstable mode compared to the continental ridge case, and therefore the second instability was able to appear on the density interface only at later times. However, after the front became unstable, its steepness relaxed, and the instability was never observed to grow to large amplitude.

\subsection{The ridge over a continental slope}

A mixture of the two behaviours described in $\S 4.1$ and $\S 4.2$ occurred when a continental ridge was positioned over a continental slope (figure $1 d$ ). During the first instability over the continental shelf the flow was identical to the flat bottom case (figure $8 a$ ). The breaking waves produced during this instability moved over the ridge and gently turned alongshore, forming the front of the new wider current that was generated by interaction of the eddies of the first instability and consequent reduction of their vorticity (figure $8 b$ ). At this stage the width of this current was similar to the width observed in the continental slope case, hence the presence of a continental slope suppressed the strong radial slowing down of the continental ridge. However, after the density interface touched the bottom and the bottom outcrop reached the edge of the continental shelf, as shown in figure 3 , the new current became unstable again and this disturbance grew to large amplitude with an initial dominant anticyclonic motion (figure $8 c$ ). The 'backwards' breaking waves spread radially outwards across 
(a)

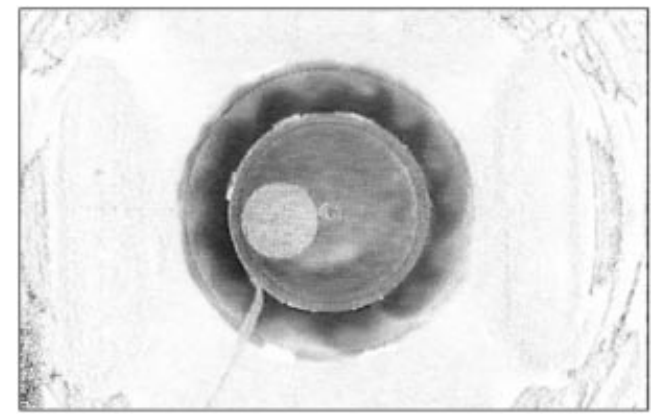

(c)

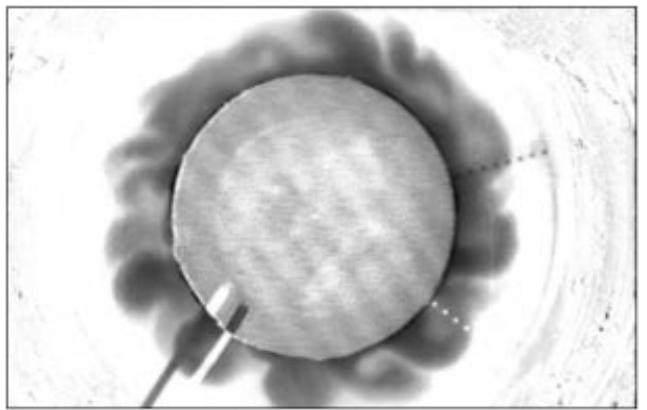

(e)

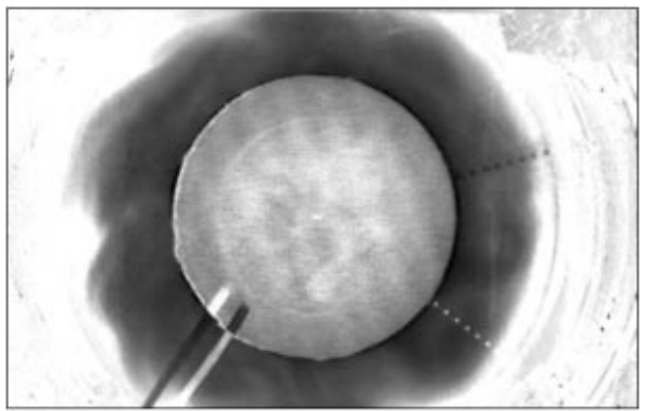

(b)

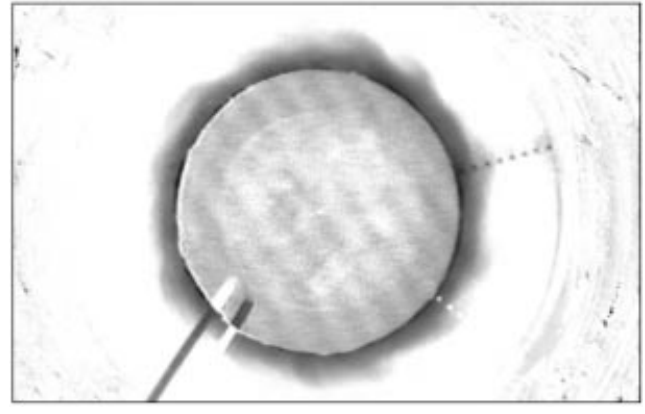

(d)

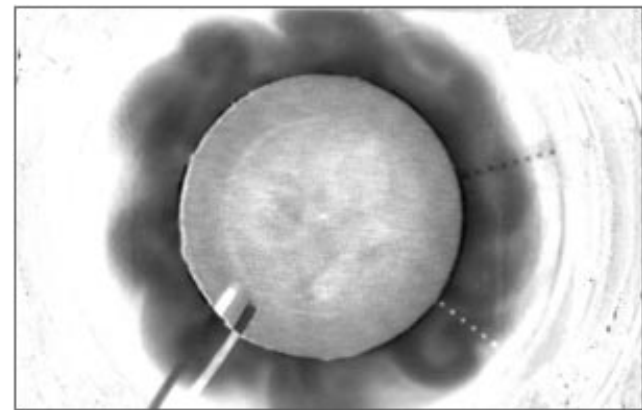

(f)

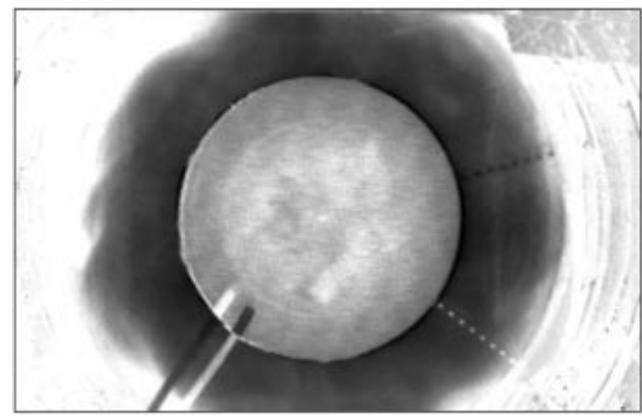

FIGURE 8. Ridge over a continental slope. A similar sequence as in figure 4 except that the current is produced over a ridge over a continental slope. The images were taken at the same times as in figure 5 and the variables values are the same as in figure 5 . The continental shelf was positioned at a high $h_{b}=19 \mathrm{~cm}$.

the slope and consequently transported ambient fluid with them. This movement stretched ambient fluid columns that started spinning cyclonically in order to conserve potential vorticity. The cyclonic motion entrained dyed fluid from the breaking waves and strong coupled vortices of opposite sign formed (figure 8d). These eddies did not detach from the current and, in a similar fashion as for the first instability, they interacted and their vorticity decreased to form an axisymmetric current (figure $8 e, f$ ). This current continued spreading radially outwards. In conclusion, the presence of the continental ridge caused the second instability to grow to large amplitude, but the continental slope constrained the radial mass transport due to the breaking waves and, through pairing of vortices that did not propagate outwards because of the continental slope, allowed a new axisymmetric current to form. 


\section{Instability over a bottom topography for large Rossby radius}

For large values of the Rossby radius of deformation of the first instability, the current width at the onset of this first instability was large compared with the width $W$ of the continental shelf, and the presence of bottom topography only slightly modified the flow structure.

The presence of a continental ridge (figure $1 b$ ) did not affect the initial outward spreading of the buoyant current over the shelf break, but it decreased the value of the depth ratio $\gamma$ once the current spread radially beyond the continental ridge. The current, at the onset of the first instability, had larger values of $L$ and $h_{1}$ than in the flat bottom case and the density interface eventually touched the bottom over the continental shelf for small values of the source depth $h_{s}$. Larger values of $L$ and $h_{1}$ were necessary in order to satisfy the criterion described in $\S 3$ that requires $F_{1}$ and $\gamma$ to be above critical values for instability to occur. The instability first appeared as wave-like irregularities on an otherwise axisymmetric current and developed into vortex pairs as described in $\S 3$. This first instability produced very long wavelengths and occurred quite close to the outer wall. Therefore, the vortex pairs touched this wall before they could interact to form a new wider current as described in $\S 4.1$. A further increase of the Rossby radius decreased the Froude number $\left(F_{1}=f^{2} L^{2} / g^{\prime} h_{1}\right)$ to such an extent that it did not satisfy the condition $F_{1} \gg F_{c}$ for the value of the depth ratio $\gamma$ of the current (§3) and, therefore, the first instability was also suppressed. Further studies in a larger tank would be useful in order to understand whether the instabilities would occur at later times, i.e. at larger current widths.

When a continental slope (figure 1c) was used, the current moved radially outward over the edge of the continental shelf where the value of $\gamma$ only slightly decreased. The values of $L$ and $h_{1}$ at the onset of instability were close to the flat bottom case and the density interface never touched the bottom on the continental shelf even after the first instability occurred. The front behaviour during the first instability was similar to that described in $\S 4.2$. However, after the current again acquired an almost axisymmetric shape, it was not perturbed by a wave-like disturbance, and it eventually reached the outer wall. This behaviour seems to support the suggestion given in $\S 4.2$ that the second instability was due to the increase in the steepness of the front. For large Rossby radius of the first instability, the density interface never became very steep. Therefore, the presence of the continental slope may be able to suppress completely the appearance of the second instability, or cause it to develop at much later times. For this topography we never observed the suppression of the first instability. Even if the Froude number $F_{1}$ became very small, the value of the depth ratio $\gamma$ was bigger than for the continental ridge case and it was still possible for the current to become unstable. The current also became unstable over a flat bottom for large Rossby radius of the first instability, confirming that the suppression of the first instability in a current over a continental ridge was due to the combination of a small value of the Froude number $F_{1}$ and a small value of the depth ratio $\gamma$ as predicted by the model described in GL.

The behaviour of a buoyant current with a ridge over a continental slope (figure $1 d$ ) was similar to the continental ridge case. In a series of experiments having increasing Rossby radius we observed first the suppression of the second instability and eventually also the suppression of the first instability. For this kind of topography the value of $\gamma$ was even smaller than for the continental ridge case, and the suppression of the instabilities was due again to the low value of the Froude number $F_{1}$. 


\section{Comparison of different topographies with the flat bottom case}

The model developed by GL for baroclinic instability with friction for the flat bottom case $(\S 3)$ agreed with their experimental results as shown in their figure 8 . They plotted the observed dimensionless wavelengths $\lambda / L^{*}$ of the growing disturbances against the Froude number $F_{1}^{*}=f^{2} L^{* 2} / g^{\prime} h_{1}^{*}$. Here $L^{*}$ and $h_{1}^{*}$ are the width and the depth of the current measured immediately prior to the appearance of the growing disturbances, and the wavelength $\lambda$, or the zonal wavenumber $k_{m}$, is calculated from the azimuthal wavenumber $n$ and the current radius $R_{0}+L^{*}$ according to

$$
\frac{\lambda}{L^{*}} \equiv \frac{2 \pi}{k_{m}}=\frac{2 \pi\left(R_{0}+L^{*}\right)}{L^{*} n},
$$

where the wavelength $\lambda$ of the second instability is non-dimensionalized with $L^{*}-W$, instead of $L^{*}$ (i.e. the $L^{*}$ in the denominator of (2) is replaced by $L^{*}-W$ for the second instability). Furthermore, the Froude number of the second instability is calculated replacing $L^{*}$ with $L^{*}-W$.

Figure 9 shows the values of the same variables as in figure 8 in GL for the experiments conducted in the present paper. Note that GL calculated the dimensionless wavelengths $\lambda / L^{*}$ using $R_{0}$ instead of $R_{0}+L^{*}$ in (2). A new definition of $\lambda$, taking into account the radius of the current immediately prior to the appearance of the growing disturbances, and a new definition of $L^{*}$ for the $F_{1}^{*}$ and to non-dimensionalize $\lambda$ of the second instability, taking in account the width of the current actively involved in the instability, were necessary in order to compare the results obtained with the first and second instability. The curves show the most unstable wavelengths at a given value of $\gamma$ from the model developed by GL. The experimental results for all different topographies and for both the first and second instabilities agree with the model developed for the flat bottom case, suggesting that the presence of topography does not have a major influence on the characteristics of the first instability and that the second instability has the same nature as the first instability. In particular the dependence of $k_{m}$ on $F_{1}^{*}$ is consistent with the stability curves of GL. In these experiments $\gamma>0.1$ and $F_{1}^{*} \gg 1$, and, therefore, the instability is likely to be predominantly baroclinic (e.g. Killworth 1980; Holland \& Haidvogel 1980). The data in figure 9 show that for the first instability the dimensionless wavelength is almost constant at $\lambda / L^{*} \approx 2$, despite the large variation of $n$ and independent of the form of the topography. For the first instability, the only small variation observed is that the flat bottom case experiments present a slightly larger non-dimensional $\lambda$ than the experiments with a bottom topography. Furthermore, the dimensionless wavelength for the first instability is smaller than for the second instability over the continental ridge or over the ridge over a continental slope. According to GL's model (solid lines in figure 9) this result can be explained by the different values of $\gamma^{*}=h_{1}^{*} / h_{2}^{*}$ (where $\left.h_{2}^{*}=H\left(R_{0}+L^{*}\right)-h_{1}^{*}\right)$ associated with the first and second instability and with different topographies. For $F_{1}^{*} \gg 1$ smaller values of $\lambda / L^{*}$ correspond to larger values of $\gamma^{*}$ as shown in figure 9. The data are consistent with stability curves since for the first instability $\gamma^{*} \approx 0.3-1.8$, which is larger than for the second instability over a continental ridge $\gamma^{*} \approx 0.3-0.4$, and over a continental ridge over a continental slope $\gamma^{*} \approx 0.3$. Finally, the second instability over a continental slope has the largest values of $\gamma^{*}\left(\gamma^{*} \approx 0.8-2.5\right)$ and gives smallest values of dimensionless wavelength as shown in figure 9, again consistent with the theoretical curves derived by GL. Thus the smaller value of $\gamma^{*}$ associated with the geometry of the current at the onset of the 


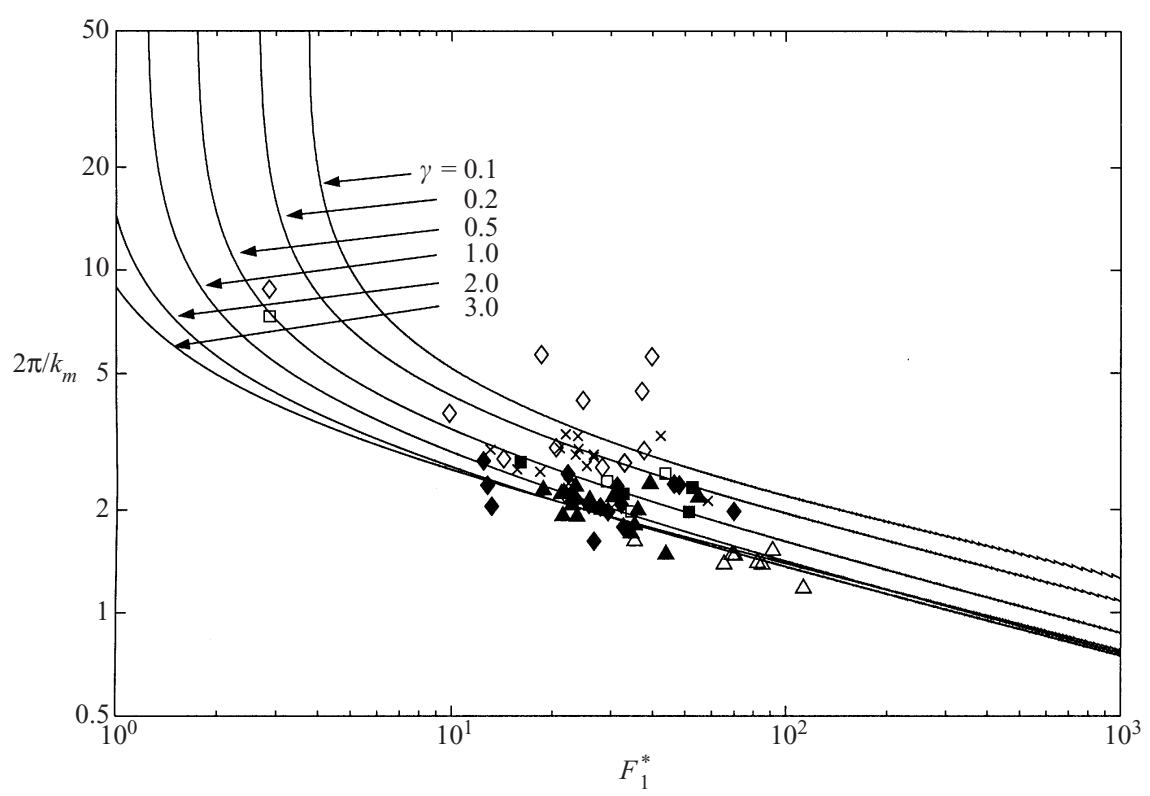

FIGURE 9. The dimensionless wavelength $2 \pi / k_{m}=\lambda / L^{*}$ as a function of the Froude number $F_{1}^{*}=f^{2} L^{* 2} / g^{\prime} h_{1}^{*}$. The data are plotted on coordinates calculated from $L^{*}$ and $h_{1}^{*}$ (width and depth of the current) measured just before instability. The curves show the most unstable wavelengths at a given value of $\gamma^{*}=h_{1}^{*} / h_{2}^{*}$ (where $h_{2}^{*}=H\left(R_{0}+L^{*}\right)-h_{1}^{*}$ ) for the model developed by GL. The filled and open symbols represents the first and second instability, respectively, over topography. Three different topographies were used: the continental ridge $(\diamond)$, the continental slope $(\boldsymbol{\Delta})$ and the ridge over a continental slope ( $\mathbf{\square})$. The flat bottom experiments are represented by the crosses (x). For the first instability $\gamma^{*} \approx 0.3-1.8$, for the second instability over a continental ridge $\gamma^{*} \approx 0.3-0.4$, over a continental ridge over a continental slope $\gamma^{*} \approx 0.3$ and over a continental slope $\gamma^{*} \approx 0.8-2.5$. The maximum error in determining $2 \pi / k_{m}$ is roughly equivalent to the symbols' vertical size, while the maximum error in determining $F_{1}$ is $\pm 25 \%$ of its value.

second instability over a continental ridge and a continental ridge over a continental slope is consistent with the observed larger dimensionless wavelength.

The large values of $F_{1}^{*}=f^{2} L^{*} / g^{\prime} h_{1}^{*}$ shown in figure 9 for both the first and second instabilities indicate that the current width at the onset of instability, and consequently the wavelength $\lambda$ of the dominant disturbance, are several times larger than the Rossby radius of deformation of the upper layer. This result is true for all the different kinds of topography and is also in agreement with GL, who argued that the instability does not occur until a sufficiently large shear is developed to overcome friction. Figure 10 shows the dependence of the wavelength $\lambda$ on the Rossby radius of deformation. A small, but significant, variation of the wavelength of the first instability with $R_{D}$ occurs when bottom topography is introduced in place of the flat bottom, the topography giving smaller values of $\lambda$ than the flat bottom for a fixed value of $R_{D}$ (already observed on figure 9). This variation gives values of the ratio between the wavelength $\lambda$ and the Rossby radius of deformation of $7<\lambda f /\left(g^{\prime} h_{1}^{*}\right)^{1 / 2}<18$, within the range given in GL. For a fixed value of $R_{D}$ the three different kinds of topographies (figure $1 b, c, d$ ) present a small variation between them for $\lambda$ of the second instability. The second instability over the ridge or over the ridge over a continental slope always has a larger value of $\lambda$ than for the second instability over the continental slope, with one exception. Furthermore, for $R_{D}>1$, the values of $\lambda$ for the second instability are slightly larger than for the flat bottom case. In addition, 


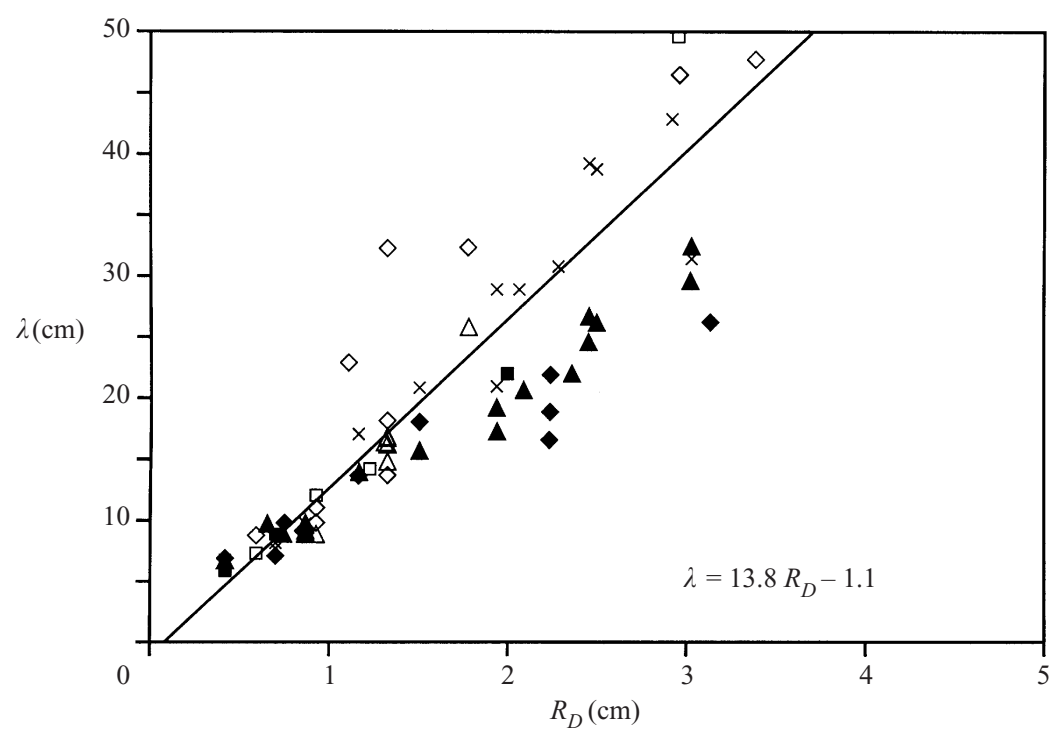

FIGURE 10. The wavelength $\lambda$ as a function of the Rossby radius of deformation of the upper layer $R_{D}=\left(g^{\prime} h_{1}^{*}\right)^{0.5} f^{-1}$. The data are fitted by least squares (solid line). The symbols are the same as figure 9 . The maximum error in determining $\lambda$ is smaller than the symbols' vertical size, while the maximum error in determining $R_{D}$ is $\pm 8 \%$ of its value.

for these values of $R_{D}$ they are larger than those of the first instability, since the width $L^{*}$ of the current for the second instability is larger but the wavenumbers $n$ observed are similar to those of the first instability. The dependence of the current width $L^{*}$ on the Rossby radius of deformation is shown in figure 11, and both the values of $L^{*}$ for the first, and $L^{*}-W$ for the second instability, are approximately 6 times larger than the Rossby radius. These values show small variations with different kinds of topography. For a fixed value of the Rossby radius the second instability over the continental slope always occurs at a larger value of $L^{*}$ than the second instability over the ridge or over the ridge over a continental slope. This differences in $L^{*}$ emphasizes the differences in the dimensionless wavelengths on figure 9 when compared to the difference in $\lambda$ in figure 10 between the values for the second instability over different kinds of topography. The dependences of $\lambda$ and $L^{*}$ ( or $L^{*}-W$ ) on the Rossby radius of deformation do not show large significant differences between the first and second instability, supporting the similar nature of these instabilities already observed in figure 9.

The behaviour of the first instability for small $(\S 4)$ and large $(\S 5)$ Rossby radii suggests that the second instability occurs when the width $L^{*}$ of the current at the onset of the first instability is smaller than the width $W$ of the continental shelf. While this is strictly true for a slope topography, the presence of a ridge allows the current disturbances to be constrained over the shelf even for values of $L^{*}$ bigger than W. If we consider $L^{*}$ as being roughly 6 times the Rossby radius (figure 11) then the second instability is expected to occur for values of the ratio $W / R_{D} \geqslant 6$ for the continental slope, while different values are expected for the continental ridge and ridge over a continental slope because, as mentioned earlier, the presence of a ridge allows the current disturbances to be constrained over the shelf even for values of $L^{*}$ bigger than $W$. Figure 12 shows the value of the ratio $W / R_{D}$ at the onset of the first instability for different topographies and these results agree with the prediction discussed above. 


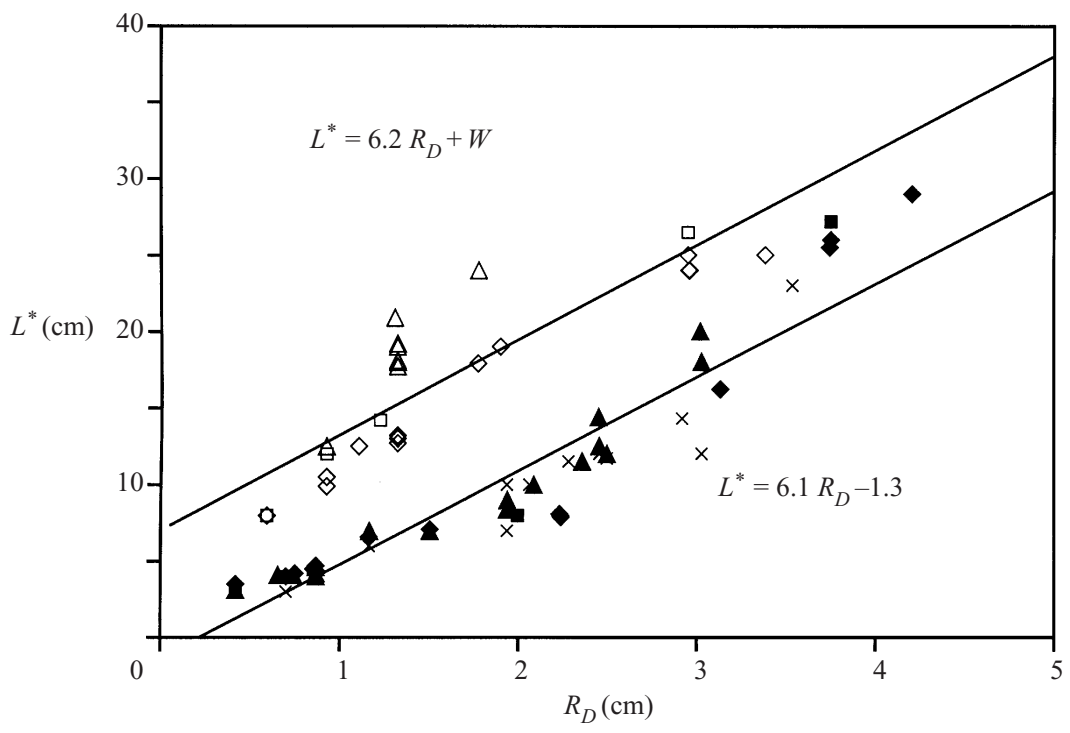

FIGURE 11. The width $L^{*}$ of the current at the onset of the first and second instability as a function of the Rossby radius of deformation of the upper layer $R_{D}=\left(g^{\prime} h_{1}^{*}\right)^{0.5} f^{-1}$. The data are fitted by least squares (solid lines). The symbols are the same of figure 9. The maximum error in determining $L^{*}$ is smaller than the symbols' vertical size, while the maximum error in determining $R_{D}$ is $\pm 8 \%$ of its value.

The second instability occurs when the ratio $W / R_{D}$ is equal or larger than $6,3,2$ for the continental slope, ridge over a continental slope and the continental ridge, respectively.

\section{Comparison of different topographies for small Rossby radius}

For the experiments with small $R_{D}$ of the first instability $\left(W \geqslant 2 R_{D}-6 R_{D}\right)$ the most striking difference in the behaviour of a buoyancy-driven current over different topographies is the radial spreading of the current. The radius $r$ of the current was determined by measuring the area $A_{c}$ occupied by the current plus the area $A_{s}=\pi R_{0}^{2}$ occupied by the cylinder of radius $R_{0}$, and assuming that this area was relocated in a circle $A_{c}+A_{s}=\pi r^{2}$, of radius $r$. Measurements were taken every $30 \mathrm{~s}$ for the first three minutes and then every minute thereafter. Figure 13 shows the increase of the outer radius $r$ with time $t$ for two different values of the Rossby radius of deformation and for all four different topographies discussed in $\S 4$. The radius $r$ is non-dimensionalized by the tank radius $R$, and time is non-dimensionalized with the rotation period $T$. The continental ridge slowed down the radial spreading of the buoyant current approximately at the ridge edge, as shown in figure 14, in which case the current had the smallest radius (for the same non-dimensional time) compared with the other topographies. However, after the current became unstable a second time (marked by the solid diamond on figures 13 and 14), the disturbances increased the radius of the current more rapidly than in the other cases and the current reached the outer wall in approximately 200 rotation periods. For the other three kinds of topography the radial spreading was approximately the same for the first 100 periods, with the current spreading beyond the width of the continental shelf. However, after the second instability in the presence of a ridge over a continental slope the current spread radially more rapidly than in the cases of a flat bottom or a continental slope 


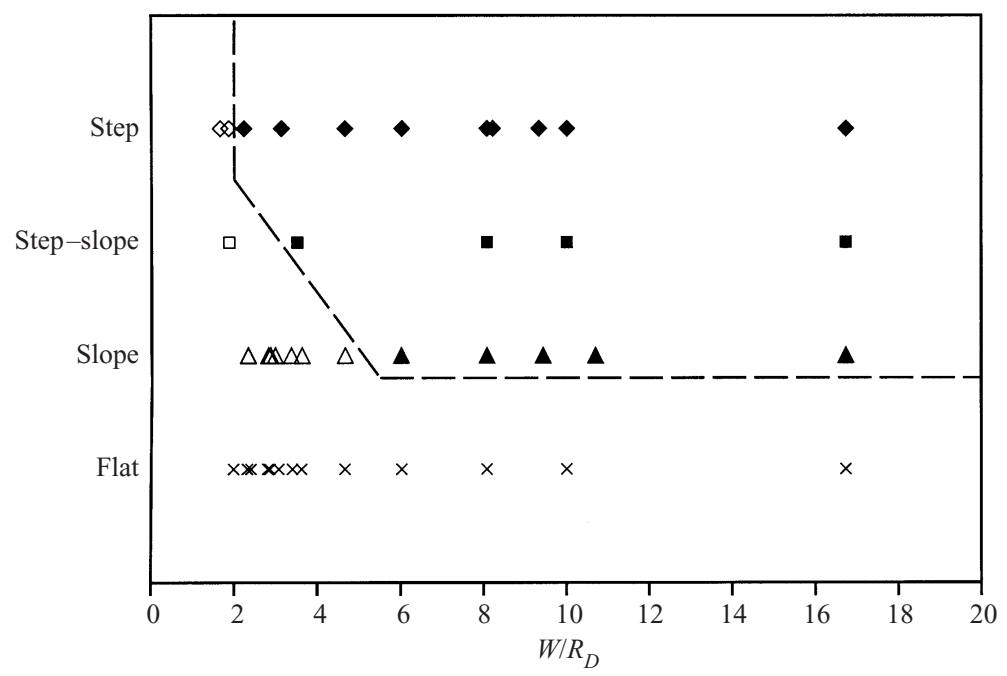

FIGURE 12. The instabilities observed (first instability only-open symbols; first and second instabilities - filled symbols) as a function of the value of the ratio of the continental shelf width $W$ to the Rossby radius of deformation $R_{D}$ at the time of onset of the first instability. Results are for the continental ridge $(\bullet)$, the continental slope $(\boldsymbol{\Lambda})$, the ridge over a continental slope $(\boldsymbol{\square})$ and the flat bottom experiments (x). The maximum error in determining $W / R_{D}$ is $\pm 8 \%$ of its value.

(the latter configuration had the lowest rate of spreading). The initially rapid radial spreading due to the ridge was diminished by the presence of the continental slope which allowed the formation and growth of a chain of eddies of alternating sign in the current. Hence, the spreading of the current over a ridge over a continental slope became smaller than for a current over a continental slope (figure 13). Both these currents (and possibly the current over a flat bottom) reached the edge of the tank after 350-400 rotation periods, almost double the time for the current over a step.

The radial spreading of the coastal current was also influenced by the value of the Rossby radius. Figure 13(b) shows that for large $R_{D}$ the rate of radial flow is consistently larger for all the four different kinds of topography before 100 rotation periods (corresponding at the initial spreading of the currents over the edge of the continental shelf). After this stage the rates of outward flow are approximately the same. Hence, for a large Rossby radius the currents reached the outer wall at slightly earlier times and their widths were always larger than the currents having a lower Rossby radius.

\section{Conclusions}

Boundary surface currents in a two-layer rotating system driven by a buoyancy source were produced in a laboratory tank. Buoyant fluid was supplied continuously and uniformly along the vertical boundary and the current increased in depth and width until it became unstable to wave-like disturbances. The flow then proceeded to became almost axisymmetric again. When topography was introduced a second instability of the buoyancy-driven current was observed.

The parameter describing the flow is the ratio of continental shelf width $W$ to the width $L^{*}$ of the current at the onset of the first instability. The values of $L^{*}$ for the first instability and $L^{*}-W$ for the second instability were not influenced by the topography and were $2-6$ times the Rossby radius (figure 11 ). Thus the parameter 

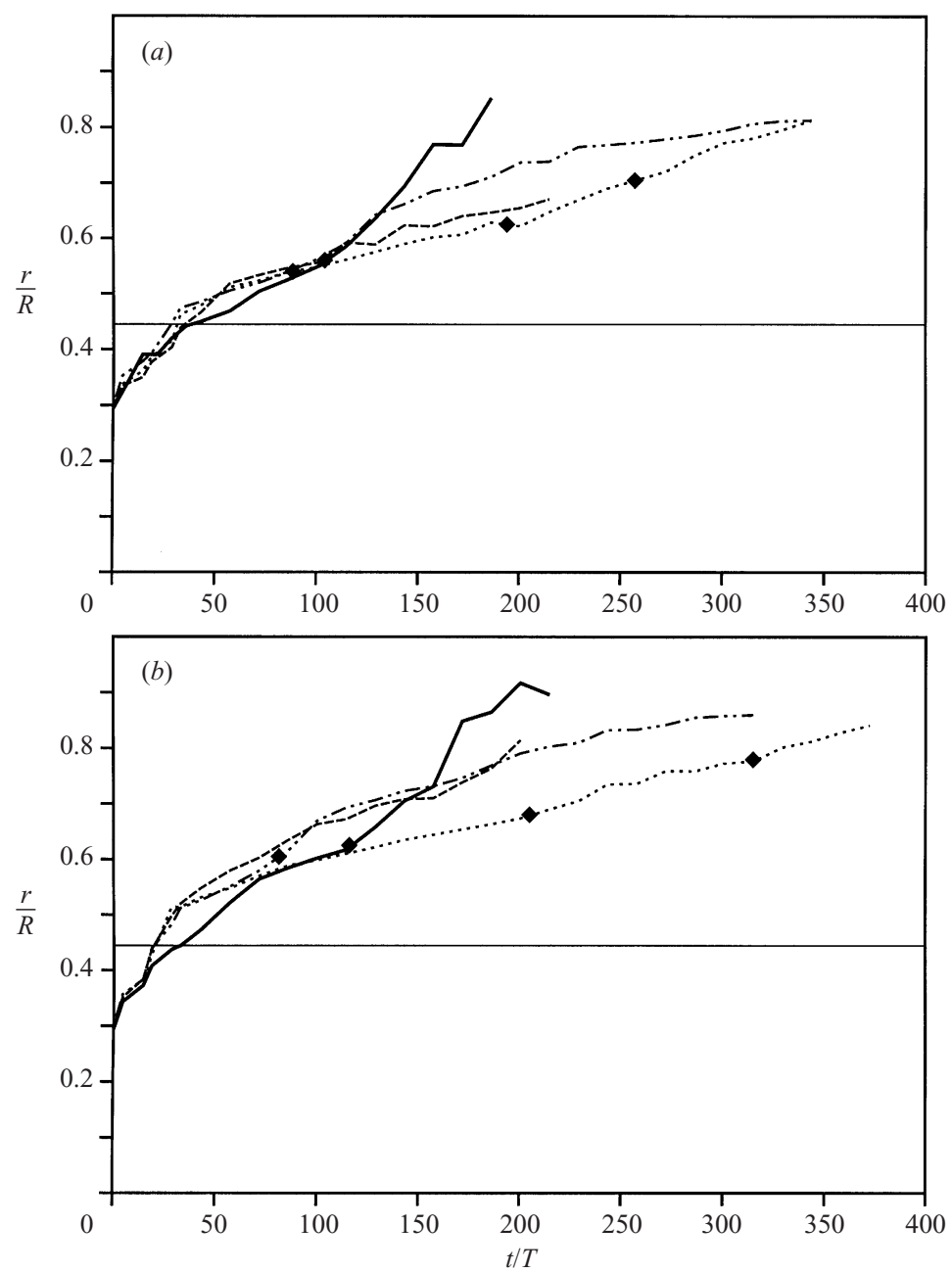

FIGURE 13. Current radius $r=L+R_{0}$, non-dimensionalized with the tank radius $R$, vs. time $t$, non-dimensionalized with the rotation period $T$, for two different values of the Rossby radius $R_{D}=\left(g^{\prime} h_{s}\right)^{0.5} f^{-1}$ (where $\left.h_{s}=3.5 \mathrm{~cm}\right):(a) R_{D}=0.9 \mathrm{~cm}$ and $(b) R_{D}=1.3 \mathrm{~cm}$. The bold solid line represents the continental ridge, the dot-dashed line the ridge over a continental slope, the dotted line the continental slope and the dashed line the flat topography. The thin solid line indicates the radius of the continental ridge. The solid diamonds mark where the current becomes unstable a second time.

describing the flow can be expressed as the ratio of the width of the continental shelf to the Rossby radius of the first instability. The second instability occurs when this ratio is larger than $2-6$ as shown in figure 12 .

For values of $L^{*} \leqslant W$, or $W / R_{D} \geqslant 2-6$, (the first instability occurring within the width of the continental shelf) the initial qualitative behaviour was not affected by the presence of topography. However, the vortex pairs produced during the first instability interacted to form a broader alongshore current that eventually became unstable for the second time to wave-like disturbances. These disturbances grew to large amplitude only for the continental ridge and the ridge over a continental slope. For a continental ridge, anticyclonic vortices formed and developed into fronts, contributing to a large radial spreading of fluid. In the case of a ridge over a 


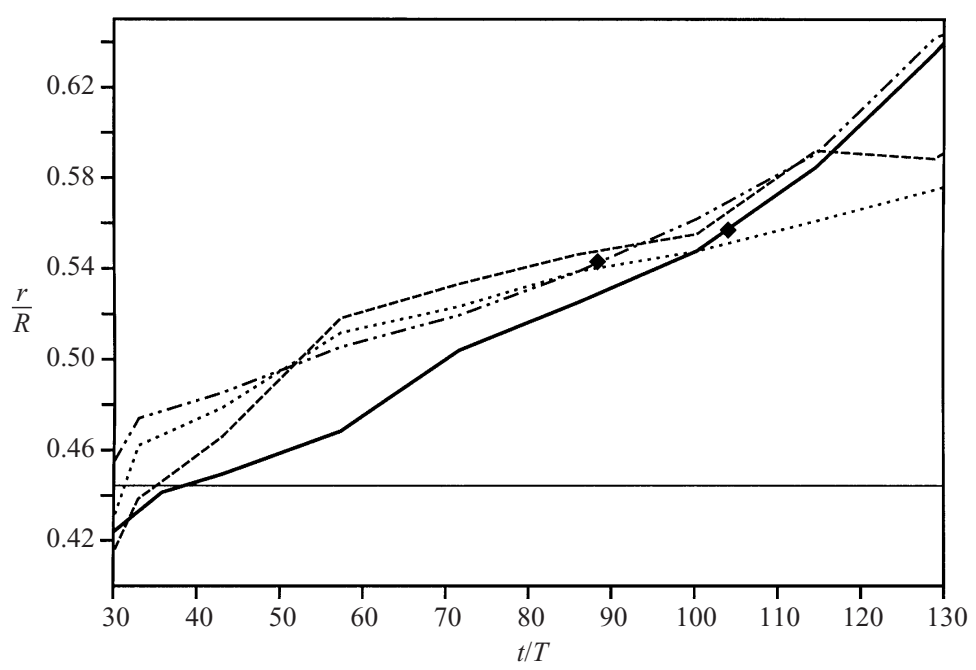

FiguRE 14. Enlargement of figure $13(a)$ between $30 \leqslant t / T \leqslant 130$ and $0.4 \leqslant r / R \leqslant 0.7$, to illustrate the behaviour of the front for the time period in which it is moving over the shelf break.

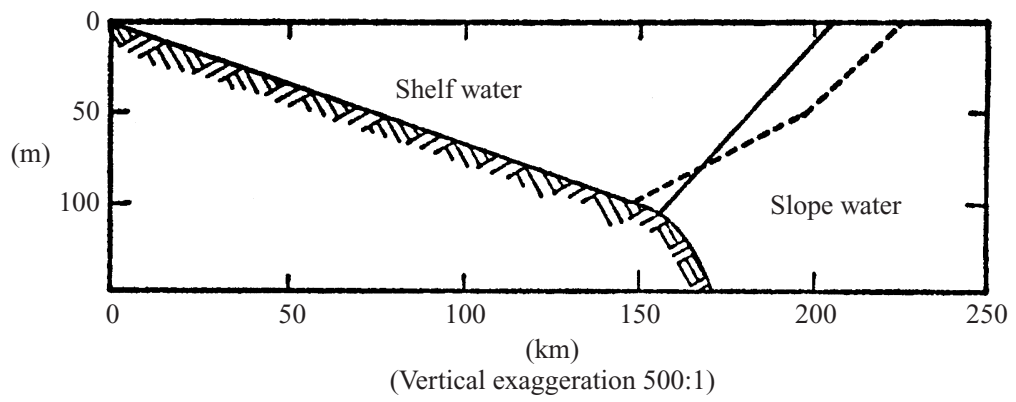

FIGURE 15. Schematic representation of change in cross-section of shelf water, winter to summer. Solid line represents the boundary in winter, dashed line in summer (taken from Wright 1976).

continental slope, the formation of a chain of vortices of alternating sign allowed the current to again acquire an axisymmetric configuration. For the continental slope on its own, the growth rate and amplitude of the disturbances were reduced such that the current became axisymmetric again.

For values of $L^{*}>W$, or $W / R_{D} \leqslant 2-6$, (the first instability occurring beyond the continental shelf) the topography did not affect the initial outward spreading of the buoyant current over the continental ridge or over a ridge over a continental slope, but affected the value of the depth ratio $\gamma$, which decreased beyond the ridge. The disturbances in this case were relatively long waves and started developing quite close to the outer wall, with the vortex pairs touching the wall before they could interact to form a new current. When increasing the Rossby radius, the value of the Froude number became smaller, and in conjunction with the smaller values of the depth ratio $\gamma$, it did not satisfy the condition $F_{1} \geqslant F_{c}$ necessary for the first instability to occur (for details see GL). Therefore, the first instability was not observed for very large values of the Rossby radius when a continental ridge or a ridge over a continental slope were used, since these gave the smallest values of $\gamma$.

For a continental slope, the current moved radially outward over the edge of the 
continental shelf where the value of $\gamma$ only slightly decreased. Therefore, we never observed the suppression of the first instability for this topography. At larger Rossby radius, even if the Froude number $F_{1}$ became very small, the value of the depth ratio $\gamma$ was bigger than for the continental ridge case and it was still possible for the current to become unstable. The current also became unstable over a flat bottom, confirming that the suppression of the instability in a current over a continental ridge or a ridge over a continental slope was due to the combination of a small value of the Froude number $F_{1}$ and a small value of the depth ratio $\gamma$ as predicted by the model described in GL.

The presence of the outer wall affected the results of the experiments with large values of the Rossby radius of the first instability. The disturbances of the current front occurred close to the outer tank wall and the further development of the instability and generated eddies was not possible. The suppression of the first instability for the largest value of $R_{D}$ should be tested in a larger tank since it would be useful to understand whether the instabilities are completely suppressed or occur at later times, i.e. at larger current widths. Further studies in a larger tank will be necessary in order to fully understand the behaviour of a buoyancy-driven coastal current for very large values of the Rossby radius of deformation.

The results obtained for a current over topography agree with the model developed by GL for a current over a flat bottom (figure 9), suggesting that the presence of a topography does not have a major influence on the characteristics of the first instability, and that the first and second instability have the same nature. A small but significant variation was found in the wavelength of the first instability, which was smaller for a current over a topography than over a flat bottom (figure 10).

At the edge of the Middle Atlantic Bight between Cape Cod and Cape Hatteras (Wright 1976; Garvine et al. 1988) the position of the front suggests an interesting relation to the experimental results obtained with a continental slope described in $\S 4.2$ and illustrated in figure 7. Frontal positions averaged over may sections suggest that the surface boundary of the front at the outer edge of the current (figure 15) moves offshore in summer, combined with a slight shoreward movement of the bottom boundary and this front is much steeper during the winter. Detachment of small vortices with diameter approximately 2-4 times the Rossby radius of deformation are associated with this front. These vortices are found more than $90 \%$ of the time from March to July, and more than $60 \%$ of the time from December to September. A possible explanation for this behaviour is that the front at the outer edge of the current becomes very steep during the winter (see figure 15) and therefore, in a similar fashion as described in $\S 4.2$, it is unstable and the disturbances grow to give the observed detached vortices between the winter and summer. In summer the front is less steep and the instability is suppressed. A similar behaviour has been observed in the experiments described in $\S 4.2$ where the disturbances also had a lengthscale 3-5 time the Rossby radius, although they were not able to grow to larger amplitude and give rise to detached vortices.

We wish to thank Ross Griffiths, Glen Gawarkiewicz and David Chapman for their suggestions and advice and for carefully reading drafts and substantially improving the clarity of the manuscript. The laboratory experiments were performed in the Department of Applied Mathematics and Theoretical Physics at the University of Cambridge. Partial support for C.C. was provided by a TMR fellowship, MAS3CT96-5017. 


\section{REFERENCES}

Cenedese, C. \& Dalziel, S. B. 2000 Concentration and depth fields determined by the light transmitted through a dyed solution. Proc. 8th Intl Symp. on Flow Visualization (ed. G. M. Carlomagno \& I. Grant). ISBN 0953399109. Paper 051. www.ode-web.demon.co.uk.

Chapman, D. C. \& Lentz, S. J. 1994 Trapping of a coastal density front by the bottom boundary layer. J. Phys. Oceanogr. 24, 1464-1479.

Condie, S. A. 1993 Formation and stability of shelf break fronts. J. Geophys. Res. 98, 12405-12416.

Dalziel, S. B. 1992 Dig Image: System Overview. Cambridge Environmental Research Consultants, Ltd. $43 \mathrm{pp}$.

FlaGg, C. N. \& Beardsely, R. C. 1978 On the stability of the shelf water/slope water front south of New England. J. Geophys. Res. 83, 4623-4631.

Garvine, R. W., Wong, K. C., Gawarkiewicz, G. G. \& McCarthy, R. K. 1988 The morphology of shelfbreak eddies. J. Geophys. Res. 93, 15593-15607.

Gawarkiewicz, G. 1991 Linear stability models of shelfbreak fronts. J. Phys. Oceanogr. 21, 471-488.

Griffiths, R. W. 1986 Gravity currents in rotating systems. Annu. Rev. Fluid Mech. 18, 59-89.

Griffiths, R. W. \& Linden, P. F. 1981 The stability of buoyancy-driven coastal currents. Dyn. Atmos. Oceans 5, 281-306 (referred to herein as GL).

Griffiths, R. W. \& Pearce, A. F. 1985 Satellite images of an unstable warm eddy derived from the Leeuwin Current. Deep-sea Res. 32, 1371-1380.

Hallock, Z. R. 1985 Variability of frontal structure in the southern Norwegian sea. J. Phys. Oceanogr. 15, 1245-1254.

Hansen, B. \& Meincke, J. 1979 Eddies and meanders in the Iceland-Faroe Ridge area. Deep-sea Res. 26a, 1067-1082.

Holland, W. R. \& Haidvogel, D. B. 1980 A parameter study of the mixed instability of idealized ocean currents. Dyn. Atmos. Oceans 4, 185-215.

Houghton, R. W., Olson, D. B. \& Celone, P. J. 1986 Observation of an anticyclonic eddy near the continental shelf break south of New England. J. Phys. Oceanogr. 16, 60-71.

Johannessen, O. M. \& Mork, M. 1979 Remote sensing experiment in the Norwegian Coastal Waters. Spring 1979. Rapport 3/79, Geophysisk Institutt, Universitetet i. Bergen.

JONES, S. 1977 Instabilities and wave interactions in a rotating two layer fluid. $\mathrm{PhD}$ thesis, Cambridge University.

JoYCE, T. M. 1977 A note on the lateral mixing of water masses. J. Phys. Oceanogr. 11, 526-540.

Killworth, P. D. 1980 Barotropic and baroclinic instability in rotating stratified fluids. Dyn. Atmosph. Oceans 4, 143-184.

LEGECKIS, R. 1978 A survey of worldwide sea surface temperature fronts detected by environmental satellites. J. Geophys. Res. 83, 4501-4522.

Linden, P. F. 1991 Dynamics of fronts and eddies. In Proc. of Enrico Fermi Summer School, pp. 313-351.

Linder, C. A. \& Gawarkiewicz, G. 1998 A climatology of the shelfbreak front in the Middle Atlantic Bight. J. Geophys. Res. 103, 18405-18423.

Orlanski, I. 1968 The instability of frontal waves. J. Atmos. Sci. 25, 178-200.

Pearce, A. F. \& Griffiths, R. W. 1991 The mesoscale structure of the Leeuwin current: a comparison of laboratory models and satellite imagery. J. Geophys. Res. 96, 16739-167575.

Pedlosky, J. 1964 The stability of currents in the atmosphere and ocean, I. J. Atmos. Sci. 21, 201-219.

Reszka, M. K. \& Swaters, G. E. 1999a Eddy formation and interaction in a baroclinic frontal geostrophic model. J. Phys. Oceanogr. 29, 3025-3042.

ReszKa, M. K. \& Swaters, G. E. $1999 b$ Numerical investigation of baroclinic instability in the Gaspé Current using a frontal geostrophic model. J. Geophys. Res. 104, 25685-25696.

Stewartson, K. 1957 On almost rigid rotation. J. Fluid Mech. 3, 17-26.

Swaters, G. E. 1993 On the baroclinic dynamics, Hamiltonian formulation, and general stability characteristics of density-driven surface currents and fronts over sloping continental shelf. Phil. Trans. R. Soc. Lond., A 345, 295-325.

Wadhams, P., Gill, A. \& Linden, P. F. 1979 Transects by submarine of the East Greenland Polar Front. Deep-Sea Res. 26, 1311-1327. 
Wright, W. R. 1976 The limits of shelf water south of Cape Cod, 1941 to 1972. J. Mar. Res. 34, $1-14$.

Yankovsky, A. E. \& Chapman, D. C. 1997 A simple theory for the fate of buoyant coastal discharges. J. Phys. Oceanogr. 27, 1386-1401. 